(2)

Literaturwissenschaft und Sozialwissenschaften 5 


\title{
Literaturwissenschaft und Sozialwissenschaften 5
}

\author{
Literatur im Feudalismus
}

Mit Beiträgen von

Wolfgang Beutin

Hubertus Fischer

Dieter Kartschoke

Irmela von der Lühe

Werner Röcke

Gerhard Schindele

Paul Gerhard Völker

herausgegeben von

Dieter Richter

J. B. Metzler Stuttgart 
ISBN 978-3-476-00292-1

ISBN 978-3-476-03038-2 (eBook)

DOI 10.1007/978-3-476-03038-2

(C) Springer-Verlag GmbH Deutschland 1975

Ursprünglich erschienen bei J. B. Metzlersche Verlagsbuchhandlung und Carl Ernst Poeschel Verlag GmbH 1975 


\section{Inhalt}

Vorwort des Herausgebers . . . . . . . . . . . . . . . . . . 1

Anmerkungen . . . . . . . . . . . . . . . 8

Dieter Richter

"Ritterliche Dichtung"

Die Ritter und die Ahnengalerie des deutschen Bürgertums $\ldots \ldots \ldots$

Anmerkungen . . . . . . . . . . . . . . . . 33

Irmela von der Lühe / Werner Röcke

Ständekritische Predigt des Spätmittelalters am Beispiel

Bertholds von Regensburg . . . . . . . . . . . . . . . . 41

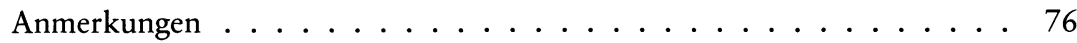

Hubertus Fischer / Paul-Gerhard Völker

Konrad von Würzburg: ’Heinrich von Kempten<

Individuum und feudale Anarchie . . . . . . . . . . . . 83

Anmerkungen . . . . . . . . . . . . . . . . . . . . 123

Gerhard Schindele

'Helmbrecht.

Bäuerlicher Aufstieg und landesherrliche Gewalt . . . . . . . . . . 131

Anmerkungen . . . . . . . . . . . . . . . . . . . . 198

Dieter Kartschoke

Weisheit oder Reichtum?

Zum Volksbuch von Fortunatus und seinen Söhnen . . . . . . . . . . 213

Anmerkungen . . . . . . . . . . . . . . . . . . . 251

Wolfgang Beutin

Psychoanalytische Kategorien bei der Untersuchung mittelhochdeutscher

Texte . . . . . . . . . . . . . . . . . . 261

Anmerkungen . . . . . . . . . . . . . . . . . . . 294

Paul-Gerhard Völker

Feudalismus als Problem materialistischer Geschichtsbetrachtung . . . . . 297

Anmerkungen . . . . . . . . . . . . . . . 332

Register . . . . . . . . . . . . . . . . 341 


\section{Vorwort}

"Ich entsinne mich « - schreibt Franz Schultz in seinen Erinnerungen an den Studienbetrieb im Berliner Germanischen Seminar um 1900 - "daß ich ein Referat über Wenzel Scherffer und die Sprache der Schlesier im 17. Jahrhundert zu halten hatte und in einer Einleitung einen zeit- und geistesgeschichtlichen Anlauf zu nehmen suchte, worin Tatsachen und Gesichtspunkte panoramisch zusammengefügt waren, wie Erich Schmidt es bisweilen in seinen Arbeiten selber tat. Aber ich fuhr schlecht. Nach der Frage ,Was soll das? rallgemeine Übersicht، zu Anfang handeln, hieß es: ,So arbeiten wir hier nicht!.« [1]

Es war die Zeit der "philologischen Disziplinen «, unter ihnen einer »deutschen Philologie «, die in der positivistisch-archivalischen Versenkung in die textualen, sprachhistorischen und überlieferungsgeschichtlichen Momente der Literatur ihr scheinbar unbezweifelbares wissenschaftliches Selbstverständnis fand: "Noch waren die Geisteswissenschaften nicht durch Methodenstreitigkeiten und grundsätzlich auseinandergehende Wege geteilt. Ein Geist herrschte, mochte man sich der Germanistik, der klassischen oder der neueren Philologie, der Geschichtswissenschaft oder der Kunstgeschichte zuwenden. Jene unsägliche Verwirrung und Trübung, die die folgenden Jahrzehnte brachten, dadurch, daß die Geisteswissenschaften die Frage aufzuwerfen sich gezwungen sahen, was sie denn eigentlich zu tun hätten, wo ihre 'Probleme lägen und wie sie anzufassen wären, blieben uns im wesentlichen noch fern, wenn auch gewisse Bestrebungen nicht übersehen werden konnten, die mit größerer oder geringerer Bewußtheit darauf abzielten, die Selbstsicherheit des philologisch-historischen Weltbildes aufzulösen. " [2]

Der objektivistisch-positivistische Wissenschaftsbegriff der philologischen Schule kommt in der Tat in nichts besser zum Ausdruck als in jenem selbstbewußten Stolz, daß es so etwas wie ein Gegenstands- und Methodenproblem der Literaturwissenschaft überhaupt nicht gäbe. "Was sie denn eigentlich zu tun hätten, wo ihre >Probleme lägen und wie sie anzufassen wären « - diese Fragen erschienen absurd. Und dies nicht deswegen, weil die philologischen Disziplinen sie für sich beantwortet hätten, sondern weil allein das Aufgreifen dieser Fragestellungen den Makel der "Unwissenschaftlichkeit « an sich trug. Der philologische Positivismus konstituierte sich auch durch den Verzicht auf Wissenschaftstheorie. 
Er konstitutierte sich damit zugleich - und dies wird häufig übersehen - durch die Absage an die wissenschaftsgeschichtliche Tradition der Germanistik in der ersten Hälfte des 19. Jahrhunderts, deren Fortführung er dennoch für sich reklamierte. Denn genau aus der Erörterung jener Fragen, was denn die Germanistik eigentlich zu tun hätte, wo ihre Probleme lägen und wie sie anzufassen wären, aus der auch politischen Reflexion auf das Gegenstands- und Methodenproblem also hatte die Literaturgeschichtsschreibung in Deutschland ihre Anfänge genommen, hatte sie sich mit Georg Gottfried Gervinus, Hermann Hettner, Robert Prutz u. a. bürgerlich-demokratischem Engagement verbunden. [3]

Die Literaturgeschichtsschreibung eines Georg Gottfried Gervinus etwa verstand sich als Literaturgeschichtsschreibung in politischer Absicht: Entstanden in der Periode der politischen Reaktion war sie - in ihrem Verständnis vom Gegenstand und der Art seiner Behandlung - wirkendes Moment jener enttäuschten und doch wachen Hoffnungen des liberalen Bürgertums auf eine demokratische Umwälzung, die mit der Verwirklichung von Freiheit und Gleichheit auch den deutschen Nationalstaat "von unten " aus schaffen sollte.

Mit der gescheiterten Revolution von 1848, der Periode des Kaiserreichs und der Epoche des Imperialismus liquidierte mit dem Bürgertum auch die bürgerliche Germanistik ihre revolutionären Traditionen. Wo sie sich "politisch « verstand, benutzte sie die Nationalidee als Vehikel von Irrationalismus, Chauvinismus und Imperialismus. Wo sie sich " unpolitisch « gab, wurde sie zum philologischen Positivismus, dessen unbestreitbare Leistungen erkauft wurden um den Preis einer politischen und wissenschaftstheoretischen Indifferenz, die sie zum bewußtlosen Werkzeug politischer Herrschaft machte.

Die "Selbstsicherheit des philologisch-historischen Weltbildes «, die der Germanist Franz Schultz in seinen oben wiedergegebenen Erinnerungen an das Jahr 1900 registriert (und in allmählicher Auflösung begriffen sieht), hat sich gerade in der Altgermanistik bis in die jüngste Vergangenheit erhalten. Noch bis vor kurzem (wenn nicht bis heute) mochte es dem Studenten, der in einem Referat »einen zeit- und geistesgeschichtlichen Anlauf zu nehmen suchte, worin Tatsachen und Gesichtspunkte panoramisch zusammengefügt waren " widerfahren, daß ihm mit einem "So arbeiten wir hier nicht! « das historische Interesse ausgetrieben wurde. Allenfalls ein geistesgeschichtliches Verhältnis zur Geschichte konnte sich legitimieren.

Denn die seit 1900 entwickelten Methoden geistesgeschichtlicher Literaturbetrachtung, von der historisch-philologischen Schule noch als "Auflösungstendenzen « empfunden, wurden - mit wechselnder und (auch lokal) unterschiedlicher Gewichtung - den philologischen Methoden einverleibt. Fand dabei die Neuere deutsche Literaturwissenschaft (im 19. Jahrhundert der Altgermanistik methodologisch und institutionell noch durchaus verbunden) jetzt ihr methodologisches Selbstverständnis eher im Kontext einer geistesgeschichtlich-idealistischen Wissenschaftstheorie, so bestimmte sich die Altgermanistik gerade durch das isolierte 
Nebeneinander von historisch-philologischen und geistesgeschichtlichen Verfahrensweisen (wobei die ersteren im allgemeinen durchaus dominierten).

Was diesen methodischen Positionen jedoch gemeinsam ist, ist ihr je verschieden negatives Verhältnis zur Geschichte: Die geistesgeschichtlichen Methoden haben ihre Beschreibungsmodelle zwar als geschichtliche deklariert; indem sie jedoch Geistesgeschichte nicht auf Sozialgeschichte rückbezogen, indem sie Geschichte nicht als dialektischen Prozeß, sondern als fortwährende Materialisation ihr angeblich übergeordneter Kategorien (Ideen, Probleme, Stile, Gattungen) zu fassen suchten, blieb ihr Geschichtsbegriff verkürzt. Irrationalistik und Formen säkularisierter Religiosität haben zusätzlich dazu beigetragen, daß jenes spezifisch deutsche Syndrom einer "Geistesgeschichte « entstand, die, auch gemessen an dem, was Geistesgeschichte tatsächlich zu erbringen vermag, durchaus insuffizient blieb. In durchaus anderer Weise hat der philologische Positivismus Geschichte für sich reklamiert: als Geschichte der textualen Fakten. Doch was hier zum Gegenstand erklärt wurde, war nicht mehr die Geschichte, sondern der Text als Summe lettristischer Materialien. Die - faszinierende - Konsistenz dieses Literaturbegriffs ist erkauft um den Preis seiner Abstraktion von Realgeschichte.

In diesem wissenschaftstheoretischen Kontext vollzog sich bis gegen Ende der Sechziger Jahre auch die Ausbildung der Deutschlehrer an den Gymnasien. Im Zuge einer wissenschaftsgeschichtlichen Entwicklung, deren (immer auch politisch legitimiertes und legitimierbares) Interesse der Nationalkultur älterer Epochen galt, im Zuge aber auch institutionell abgesicherter Ordinarienherrschaft nahm bis vor kurzem die Beschäftigung mit Literatur und Sprache des Mittelalters die Hälfte eines Germanistikstudiums ein. Das Übersetzen aus dem Gotischen, dem Althochdeutschen und dem Mittelhochdeutschen, dazu Sprachgeschichte, Etymologie, Textkritik und Editionskunde waren die bevorzugten Übungsfelder altgermanistischer Studientätigkeit. Die Diskrepanz zwischen dieser Studientätigkeit und der Berufstätigkeit der künftigen Lehrer war nur allzu evident (wenngleich die Altgermanistik nur ein besonders krasses Beispiel der Inkohärenz von Germanistikstudium und schulischer Berufstätigkeit war). Es ist in der Tat schwierig, diesen Sachverhalt gesellschaftlich befriedigend zu erklären - sei es von einem Begriff möglicher »Funktionalität « oder tatsächlicher »Dysfunktionalität « der Ausbildung her. Immerhin wurde bereits 1882 der Sprachunterricht im Mittelhochdeutschen an den preußischen Gymnasien abgeschafft - dies übrigens gegen den Protest der Philologenversammlung und universitärer Fachkoryphäen, die in dieser Gymnasialreform ein bedenkliches Zugeständnis an sozialistische Kräfte sahen, die Wissenschaftlichkeit der Ausbildung in Gefahr wähnten und so Konrad Burchdach (1886) - damit ein Bollwerk gegen »das lauernde Gespenst der sozialen Revolution " gefallen sahen. [4]

Protest gegen den altgermanistischen Studienbetrieb kam seit Mitte der Sechziger Jahre zunächst aus den Reihen der Studentenbewegung. Doch die Parole "Schafft die (Alt-)Germanistik ab! « war nicht einfach liquidatorisch. Sie zielte auf 
die Politisierung der Studientätigkeit, auf Einsicht in die Bedingungen des gesellschaftlichen Zusammenhangs von Universitätsbetrieb und politischer Herrschaft. "Wir haben die Gesetze der zweiten Lautverschiebung gelernt, während andere die Notstandsgesetze verabschiedeten " - so faßte Peter Schneider auf einem teachin vor Berliner Studenten 1967 diese Kritik pointiert zusammen. [5] Auch der Hinweis auf die Diskrepanz von Studientätigkeit und Berufstätigkeit zielte nicht auf die Forderung nach blanker Anpassung der Studieninhalte an eine immer schon vorgegebene und unveränderbare Berufspraxis, sondern auf die politische Neubegründung von Studium und Schulpraxis.

Die politische Forderung nach Abschaffung der (Alt-)Germanistik ging freilich unter im Beifall der Herren Bildungsplaner, die aus dieser Forderung Kapital schlugen. Die kapitalistische Bildungsreform hat sich daran gemacht, den "Wildwuchs " zu beschneiden, die Ausbildungsinhalte und -formen einer "Verwertbarkeit " anzupassen, die die gesellschaftlichen Emanzipationsbedürfnisse der Betroffenen negiert und orientiert ist an einer von der "Logik des Kapitals « bestimmten Qualifikationsstruktur der Arbeitskräfte und der Aufrechterhaltung von politischer Herrschaft.

Was da auf der Strecke bleiben soll, ist das bestimmte historische Interesse und mit ihm das Interesse an einer veränderten und veränderbaren Aneignung sozialer Wirklichkeit - beides vermittelbar über die Beschäftigung mit Literatur und Literaturgeschichte. Und in der Tat zeichnen sich die neueren Studienreformmodelle durch die Zurückdrängung literaturwissenschaftlicher Ansätze auf die Spielwiese einer »musischen Bildung " aus, wo allenfalls kompensatorische Rekreationsübungen stattfinden.

Die Altgermanistik hat auf die Abschaffungsforderungen mit kläglicher Hilflosigkeit reagiert. Sie verteidigte eine verlorene Stellung nach der anderen (zunächst das Gotische, dann das Althochdeutsche, im Moment das Mittelhochdeutsche) - um sie dann, gezwungen durch den Druck der Kultusbürokratie zwar grollend aber gehorsam aufzugeben. Sie hat es bislang im allgemeinen nicht verstanden, offensiv und unter radikal selbst- und gesellschaftskritischer Preisgabe unhaltbarer Positionen ein neues wissenschaftliches, politisches und berufspraktisches Selbstverständnis zu entwickeln. Sie hat sich mancherorts wiederum auf eine "deutsche Philologie « zurückgezogen, dezimiert um den ehemals erzwungenen Massenzulauf der Studenten (die heute nur noch, widerwillig wie ehedem, ein bis zwei Pflichtkurse durchlaufen). Sie hat sich auf diese Weise wissenschaftstheoretisch und organisatorisch in ein Ghetto hineinmanövriert, was institutionell etwa in der "Dreiteilung " des Faches Germanistik in "Neuere deutsche Literatur Sprachwissenschaft - Mediävistik " (so die Rahmenrichtlinien der Kultusministerkonferenz) zum Ausdruck kommt. Denn was hier als "Mediävistik « ("Altgermanistik «, "Ältere deutsche Abteilung «) firmiert, behauptet seine institutionelle Eigenexistenz gegenüber "Neuerer deutscher Abteilung " und "Sprachwissenschaft" durch die (angebliche) Besonderheit mediävistischer Methoden. Nichts 
jedoch rechtfertigt diese angebliche methodologische oder gegenstandsspezifische Besonderheit der Altgermanistik. Denn soweit sprachhistorische Methoden gemeint sind, verbinden sie diese mit der historischen Sprachwissenschaft. Soweit es um literaturgeschichtliche Methoden geht, teilt sie diese mit der Literaturwissenschaft. Textkritik und Editionskunde sind Verfahrensweisen auch anderer Wissenschaften. Die angebliche "Besonderheit " der Mediävistik erscheint angesichts dieser Sachverhalte als Versuch einer institutionellen Rettung der eigenen Disziplin.

Die Germanistik hat sich inzwischen in der ihr auferlegten Dreiteilung - vorläufig - eingerichtet. In der Praxis hat die Altgermanistik einige Arbeitsbereiche (etwa die vergleichende Sprachforschung und die Etymologie) an die allgemeine Linguistik abgegeben oder sie versucht sich als "historische Ergänzung « strukturaler Sprachbetrachtung zu profilieren. Die an einigen Universitäten praktizierte Wahlmöglichkeit zwischen Linguistik und Altgermanistik ist der institutionelle Ausdruck dieser Entwicklung. Schließlich war es auch die Altgermanistik, die seit etwa 40 Jahren das "Eindringen " strukturaler Betrachtungsweisen in die deutsche Sprachwissenschaft verhindert hat. In anderen Fällen profiliert sich die Altgermanistik weiterhin als Philologie, anknüpfend an die langen Traditionen von Übersetzungskunde, Textkritik und Editionstechnik.

Die Frage der schulpraktischen Relevanz solcher Tätigkeiten wird dabei nur zögernd gestellt. Soll sie wenigstens ansatzweise beantwortet werden, bedarf es eines neuen, historischen Selbstverständnisses dieser Wissenschaft.

Die Autoren der hier zusammengestellten Beiträge gehen auf unterschiedliche Weise an ihre Gegenstände heran. Im Zentrum stehen einige Werk-Interpretationen: Die Verfasser verstehen sie als Versuch, sozialgeschichtliche Verfahrensweisen an Literatur zugleich zu entwickeln und zu erproben, was (auch in Anbetracht fehlender Vorarbeiten) immer auch das Problem einer werkgerechten Vermittlung von Sozialgeschichte aufwirft. Der Beitrag von Wolfgang Beutin bemüht sich um die Einbeziehung von psychoanalytischen Katgegorien in die historische Werkinterpretation: Die besonderen Schwierigkeiten dieser notwendigen Themenstellung rühren nicht nur vom Gegenstand, sondern auch daher, daß sowohl die Diskussion um den literaturtheoretischen Beitrag der Psychoanalyse als auch um das Verhältnis von Psychoanalyse und historischem Materialismus auf der allgemeinen Ebene noch so wenig fortgeschritten ist. Der Aufsatz von Dieter Richter versucht darzustellen, daß und wie die Beschäftigung mit den literarischen Gegenständen zugleich die Geschichte ihrer Behandlung einschließen muß. Paul Gerhard Völker endlich bemüht sich um den Begriff des Feudalismus als Problem materialistischer Geschichtsbetrachtung.

Ungeachtet dieser unterschiedlichen Themenstellungen und Herangehensweisen darf, vor dem Hintergrund der vorhergehenden Überlegungen, der Konsens zwischen den einzelnen Verfassern etwa folgendermaßen umrissen werden:

1. Ausgehend von der Kritik am bisherigen Selbstverständnis der "Altgermani- 
stik « wird die ältere deutsche Literatur begriffen als Teil der einen deutschen Literatur, die vom 8. Jahrhundert bis zur Gegenwart reicht. Die Verfasser gehen also von einem literaturgeschichtlichen Selbstverständnis der "Mediävistik « aus. Werke der mittelalterlichen oder frühneuzeitlichen Literatur sollen prinzipiell mit den gleichen Erkenntniszielen und den gleichen Verfahrensweisen zum Gegenstand der Untersuchung werden, wie Werke der neueren deutschen Literatur. Wo der zeitliche Abstand, die Unsicherheit der Textüberlieferung oder die Fremdheit des sprachlichen Materials dazu zwingen, werden die - wichtigen und unverzichtbaren! - Methoden der Philologie (Überlieferungsgeschichte, Textkritik, Übersetzungskunde, historische Wortbedeutungslehre etc.) hilfswissenschaftlich benutzt - wie dies im übrigen in analoger (wenn auch im einzelnen unterschiedlicher) Weise auch für die Werke der neueren deutschen Literatur zu gelten hat.

Die Werke mittelalterlicher Autoren werden also nicht - wie dies in der Tradition der »deutschen Philologie « bis heute üblich ist - als Übungstexte für das Übersetzen aus dem Mittelhochdeutschen oder für das Erlernen textkritischer Verfahren benutzt, sondern als literarische Zeugnisse einer historischen Epoche interpretiert.

2. Die Verfasser versuchen, einen spezifisch sozialgeschichtlichen Begriff von Literatur auf der Basis einer materialistischen Geschichtsauffassung zu entwikkeln. Gegen die Tendenzen von Formalisierung und Enthistorisierung der Literaturbetrachtung ebenso wie gegen die Praktiken eines geistesgeschichtlichen Reduktionismus verstehen sie Literaturgeschichtsschreibung als spezifisches Moment einer umfassenden Wissenschaft von Geschichte und Gesellschaft. Sie orientieren sich dabei an einem materialistischen Begriff von Geschichte, wie ihn Marx in der Deutschen Ideologie entwickelt hat: "Diese Geschichtsauffassung beruht also darauf, den wirklichen Produktionsprozeß, und zwar von der materiellen Produktion des unmittelbaren Lebens ausgehend, zu entwicklen und die mit dieser Produktionsweise zusammenhängende und von ihr erzeugte Verkehrsform, also die bürgerliche Gesellschaft in ihren verschiedenen Stufen, als Grundlage der ganzen Geschichte aufzufassen und sie sowohl in ihrer Aktion als Staat darzustellen, wie die sämtlichen verschiedenen theoretischen Erzeugnisse und Formen des Bewußtseins, Religion, Philosophie, Moral etc. etc., aus ihr zu erklären und ihren Entstehungsprozeß aus ihnen zu verfolgen, wo dann natürlich auch die Sache in ihrer Totalität (und darum auch die Wechselwirkung dieser verschiedenen Seiten aufeinander) dargestellt werden kann. « [6]

Der "Mittelalter «-Begriff wird, gegen die übliche geistesgeschichtliche Verkürzung, sozialgeschichtlich fundiert: Es geht um die Epoche des Feudalismus, aus der sich die bürgerlich-kapitalistische Gesellschaft prozeßhaft entwickelt. Ein solcher sozialgeschichtlich fundierter Literaturbegriff zielt nach Meinung der Verfasser nicht darauf, literarische Werke (als Momente des gesellschaftli- 
chen Bewußtseins) im Sinne eines naiven widerspiegelungsästhetischen Abbildrealismus als bloßen Reflex, sondern vielmehr als wirkendes Moment der materiellen Lebenstätigkeit der historischen Subjekte zu interpretieren. Die literarischen Werke sollen nicht als sozialgeschichtliche Belegstellenkataloge gelesen werden, sondern als spezifische Aneignungsformen geschichtlich-gesellschaftlicher Verhältnisse.

3. Die materialistische Betrachtungsweise geht nicht den linearen Weg der historischen Chronologie. Sie geht vielmehr den geschichtlichen Weg im Krebsgang. Ihr Ausgangspunkt ist die Gegenwart; ihr Ziel ist, zu erklären, wie sich die gegenwärtigen Verhältnisse aus den vergangenen herausgebildet haben und welche Momente der Überwindung beide enthalten.

Von daher ist auch die historische Akzentuierung der einzelnen Beiträge zu verstehen. Sie beschäftigen sich vorzugsweise damit, darzustellen, wo und in welcher Weise im Schoß der feudalen Gesellschaft die Tendenzen ihrer Auflösung und Überwindung sich andeuten. Dem »späten Mittelalter « und der "frühen Neuzeit « kommt daher ein besonderes Gewicht zu. Und bis in die Periode der als "klassisch " geltenden mittelalterlichen Literatur wird dieser Ansatz verfolgt. Kommt es doch dem Materialisten nicht darauf an, die harmonische Totalität einer klassischen Epoche zu feiern, sondern darauf, diese aufzulösen in den dialektischen Prozeß der Geschichte.

Hat die traditionelle bürgerliche Literaturgeschichtsschreibung zum Mittelalter ihr Augenmerk vor allem darauf gerichtet, die Konsistenz und Kontinuität feudaler Normen und Werte zu dokumentieren, so versuchen die Verfasser umgekehrt, den Spuren vorwärtsweisender historischer Momente nachzugehen. An der mittelhochdeutschen Literatur läßt sich zeigen, wie im Zuge der Entstehung der Städte, fortschreitender gesellschaftlichen Arbeitsteilung und der Entfaltung der Ware-Geld-Beziehungen feudale Ordnungen brüchig werden und bürgerliche Verkehrsformen sich entwickeln.

4. Der Feudalismus und seine Ideologie sind nicht mit dem Ende des »Mittelalters « verschwunden. Gegen ein mechanisches Schema vom Auf- und Abstieg der Klassen und der linearen Entwicklung der Epochen ist auf einem dialektischen historischen Denken zu bestehen. Dies bedeutet zum einen, zur Kenntnis zu nehmen, daß Momente eines feudalen Bewußtseins auch die Epoche der bürgerlichen Gesellschaft durchziehen - gerade in Deutschland, wo sie im Zuge verhinderter bürgerlich-revolutionärer Traditionen bis ins 20. Jahrhundert hinein eine Rolle spielen. Es bedeutet zum andern, sich von der Vorstellung zu lösen, als wären die Ideen, Forderungen und Träume, die sich - wie gebrochen auch immer - in Literatur vorbürgerlicher Epochen ausdrücken, in der bürgerlichen Gesellschaft immer schon widerspruchsfrei realisiert.

Durchaus kontrovers ist für die Autoren dieses Bandes die Frage, wieweit eine solche neue Altgermanistik für Studenten des Faches nicht nur einen möglichen, sondern einen notwendigen Beitrag im Rahmen der Deutschlehreraus- 
bildung leistet. In dieser allgemeinen Form kann die Frage offenbar ohnehin nicht beantwortet werden. Hier sind Erfahrungen zu sammeln, ist die wissenschaftliche Entwicklung des Fachs und sind die Ausbildungsbedürfnisse der Studenten zu berücksichtigen. Einen Materialbeitrag für diese Auseinandersetzungen will dieser Band darstellen. Es geht den Autoren nicht darum, die Altgermanistik zu retten. Es geht ihnen darum, aufzuzeigen, wie die Beschäftigung mit ihr nützlich sein kann.

Im Juni 1974

Dieter Richter

\section{Anmerkungen}

1 Franz Schultz, Berliner germanistische Schulung um 1900. In: Das Germanische Seminar der Universität Berlin. Festschrift zu seinem 50jährigen Bestehen. Berlin/Leipzig 1937, S. 19 ff. Nach: Materialien zur Ideologiegeschichte der deutschen Literaturwissenschaft, hg. von Gunther Reiß, Bd. 1, Tübingen 1973, S. 53.

2 ebenda, S. $50 \mathrm{f}$.

3 Vgl. Bernd Hüppauf (Hg.), Literaturgeschichte zwischen Revolution und Reaktion. Aus den Anfängen der Germanistik 1830-1870, Frankfurt 1972.

4 Konrad Burdach, Über deutsche Erziehung. In: K. B., Vorspiel, Bd. 1, Halle 1925, S. 23.

5 Peter Schneider, Wir haben Fehler gemacht. In: P. S., Ansprachen, Berlin 1970, S. 10.

6 Karl Marx, Die Deutsche Ideologie. In: Marx-Engels-Werke, Bd. 3, S. $37 \mathrm{f}$. 